\title{
Large Eddy Simulation of Shock Boundary Layer Interaction along with Wall Effects
}

\author{
Sravan kumar Kota ${ }^{1}$, Dr. Rubens Talukder ${ }^{2}$ \\ ${ }^{1}$ Project Manager Department of Aerospace and Mechanical, Samhams Technologies, Chennai, India \\ ${ }^{2}$ Chief Executive Officer of Nexeinc, Canton, Michigan, USA \\ Email id : ${ }^{1}$ sravankumarkota88@gmail.com, ${ }^{2}$ info@ nexeltinc.com
}

\begin{abstract}
It's really arduous work to simulate the shock wave interaction with boundary layer through high computational domains such as Large Eddy Simulation (LES), Direct Numerical simulation (DNS), Detached Eddy Simulation (DES) and so forth. This work comprise about numerical and computational analysis of supersonic flows where the Shockwave and Boundary layer interaction happens. Such flows uncover the presence of complex components, which should be painstakingly examined for the effective structure of Propulsion and Aerodynamic frameworks. Though, it has several flaws and efforts to acquire appropriate and effective results, the results are like precise and efficient to compare with real life experiments. In this paper the high performance computational simulation approach i.e., Large Eddy Simulation approach has used to detect, reveal and scrutinize the physical flow phenomena of shock boundary layer interaction mechanism over ObliqueImpingement (O-I) model with mach number (M) 2.7 and Reynolds number of 4300. The 3D flow structure is seen as instigated by the cleared SWBLI shaped on the sidewalls. The area of the endpoint of the episode shock close to the sidewall is restricted by a sweepback impact, permitting the meaning of an entrance Mach number $\mathrm{M}$ that is appeared to relate well with the spanwise degree of the centre stream. The acquired large-eddy simulation results have given a reasonable and exact proof of the essential adverse flow and the auxiliary detachment flow being basically tempestuous. Additionally, simulations uncover the nearness of such flow properties for the most part close to the shock foot and inside the distribution bubble. The wall effects are also studied along with sub physical characteristics such as adverse pressure gradients, interaction and re-attachment region, surface pressure distribution, expansion fan and so on. In the nutshell, the results are then compared with different angle impingements viz. 9 and 12 Degree. Here, the Tecplot360 adhere a critical part for interfacing the post processor results and visualizing them through various plots, slices and frames.
\end{abstract}

Keywords: Turbulence Models, Large Eddy Simulation, Shock Wave, Boundary Layer, High Performance Computation (HPC) Models.

\section{Introduction}

The cooperation of shock waves including boundary layers is fundamental flow elements wonder that has both principal and reasonable significance. From the designing perspective, this issue can affect airplane or rocket execution, what's more, regularly prompts incredibly bothersome impacts, for example, drag rise, gigantic stream detachment, shock instability, and high divider heating. From the principal perspective, this wonder speaks to one of the least difficult flow designs yielding a solid viscous/inviscid connection, and is along these lines a perfect experiment for Navier-Stokes solvers [4].
SWBLI is one of the gigantic areas in Hypersonic Aerodynamics, moreover critical are to cover for all intents and purposes all the vehicles, reentry cases, and space related vehicles. Also, it has advanced numerous extent of supersonic inward \& external streams, moreover, it has seemed to show a difficult smoothed out-merging of inviscid and viscous impacts. Therefore, entire SWBLI activated tarpid pressure disaster outwardly of transonic aerofoils as well as wings is a noteworthy wellspring of wavy drag [2]. Likewise, novel stream control procedures have additionally been proposed to manage the SWBLI-prompted 
decrease in delta productivity. Notwithstanding the advancement accomplished so far in various regions identified with SWBLI, there are as yet numerous inquiries unanswered, for example, the degree of demonstrating required to catch the key material science in SWBLI for designing utilizations [1].

To smooth out the issue, most computational, numerical and experimental works on SWBLI have focused on alike, quasi, and (semi-2D) interactions, where as spanwise equality is acknowledged. Regardless, with the assistance of upper-steadiness at both computational and numerical strategies, including the velocity stream affiliation and sensitive qualities of the disconnected SWBLI field, are gotten by semi 2D proliferations. In any case, it ought to be noticed that a run of the mill 2D SWBLI can possibly occur inside a restricted district for useful utilities when sidewalls are available, for example, in air-breathing supersonic and hypersonic airplane admissions. In any event, for air stream tests when estimations are normally taken in the air stream focal area, sidewall impacts are unavoidable [3].

Numerical recreations can give nitty gritty immediate stream structures and factual data, and assist to uncover instruments in back of the three-dimensionality. Nonetheless, ordinary RANS approaches can't foresee such complex streams, remembering solid detachment and an auxiliary vortex for the corner. Also, the solid cleared SWBLI, which has a blended kind division bubble is likewise hard for Reynolds model approaches. With quick increments of PC execution, it is attainable to organize high computational models at modest and average Reynolds numbers, individually, producing the adjacent wall results into considered [2].

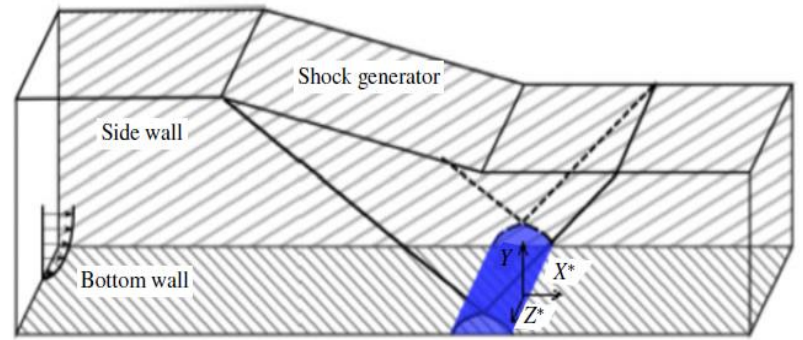

Figure 1: Sketch of the domain used for the simulations

\section{Types of SWBLI:}

In SWBLI there are Five essential interactions can happen at twodimensional streams. These happen when there is:

--an oblique impingement-shock

reflection(2-D and 3-D)

_ a ramp flow(2-D and 3-D)

- a normal shock(2-D)

_ an imposed pressure jump(2-D and 3-D)

- an oblique shock induced by a forward-

facing step(2-D and 3-D)

In a slanted shock reflection at a level surface (Fig:1), the moving toward supersonic progression of Mach number undergoes a diversion through episode stun. For the downstream stream to stay corresponding to the divider,Stun designs like this happen inside a supersonic airadmission of the blended pressure type or at the effect of the stun produced by any deterrent on a close by surface.

\section{Interaction without Separation}

The joint effort coming about on account of the impression of a corner to corner stagger wave from a wild breaking point thickness is embellished by the picture portrayal in Fig. 2. A likewise formation would be obtained for a normal breaking point layer, yet the motion level of the collaboration space would be progressively essential. The stream field affiliation is spoken to in Fig. 2. Scene daze (C1) entering the rotational inviscid part of the 
cutoff layer, where it consistently turns due to close by Mach number reduction. Correspondingly, the power incapacitates and vanishes completely when it shows up at the breaking point layer sonic line. At the same time, the weight rise through (C1) is experienced upstream of where the scene stagger would have influenced the divider without a breaking point layer. This upstream-sway wonder is overwhelmingly an inviscid framework; the weight surge realized through paralyze is passed by upstream by the subsonic bit of the cutoff layer. This prompts a isolation of the divider force dispersal on a division on the solicitation for the cutoff region thickness, differentiated and the totally inviscid-stream game plan. As showed up in Fig. 2. For this circumstance, the gooey (or veritable) game plan doesn't leave far from the totally inviscid course of action. Speaking to the gooey effect would be a basic modification to an answer that is starting at now close to this present reality. Such direct should be a weak collaboration process as in the stream is influenced fragile by thick effects. The composition of cutoff layer subsonic zone has experienced by the outer flow stream, that includes the critical bit of the breaking point region if the stream is stormy. It influence as a slope inciting pressure waves $(\eta)$ which blend to shape the bended paralyze $(\mathrm{C} 2)$. The opacity of the subsonic region relies upon the speed movement; along these lines, an all the more full profile - which has an increasingly thin subsonic domain - similarly has a tiny upstream-sway span. Moreover, a cutoff stream model along with a little speed lack have a great power and, as such, progressively unmistakable assurance from the prevention presented by a hostile weight edge.
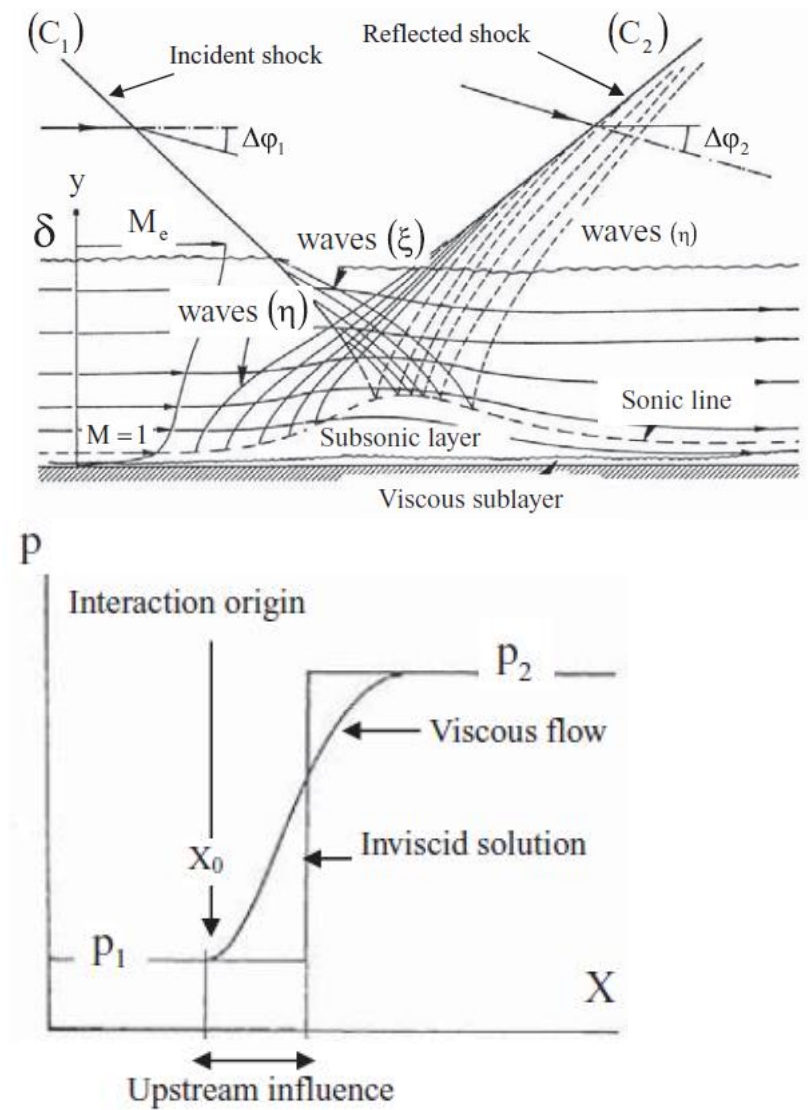

Figure 2 :Schematic physical phenomenon of interaction without separation

\section{Separation Caused by an Incident Shock}

Boundary thickness in a stream inside which the stagnation pressure diminishes when moving toward the divider and where - at any rate, for tiny separations it tends to be viewed as consistent along every smooth out. The phenomenon of this stream is represented in Figure.3. However, over the downstream of division point $\mathrm{S}$ is a recycling 'bubble' stream limited by a partitioning smooth out (S), which isolates the recycling stream from the stream gushing from upstream to downstream 'vastness'. The smooth out (S) begins at bifurcation place $S$ and closures at merged at point $\mathrm{R}$. Because of the activity of the solid blending occurring in the isolates shear region radiating from S,vitality move happens from the external rapid stream towards the isolated area. As 
a result, the speed Us on the separating smooth out (S) consistently increments until the soar related with merge procedure begins. Transmitted stun (C4) enters the isolated gooey stream, where it is reflected as an extension wave in light of the fact that there is a close steady weight level in the air pocket. This stun design is depicted in more detail in an ensuing area. As appeared in Fig. 3, the divider pressure dissemination at first shows a precarious ascent, related with partition, trailed by a level regular of isolated streams. A second, increasingly dynamic weight rise happens during reattachment. In this circumstance, the flow field structure is particularly not quite the same as what it would be for the absolutely different case than viscous, and the stun reflection is supposed to be a solid gooey inviscid association. This implies gooey impacts must be completely viewed as while foreseeing the stream. They no longer include a straightforward change in accordance with an effectively close right inviscid arrangement, yet they have a focal job in setting up the arrangement. It is obvious that there has been a progressive system inversion.

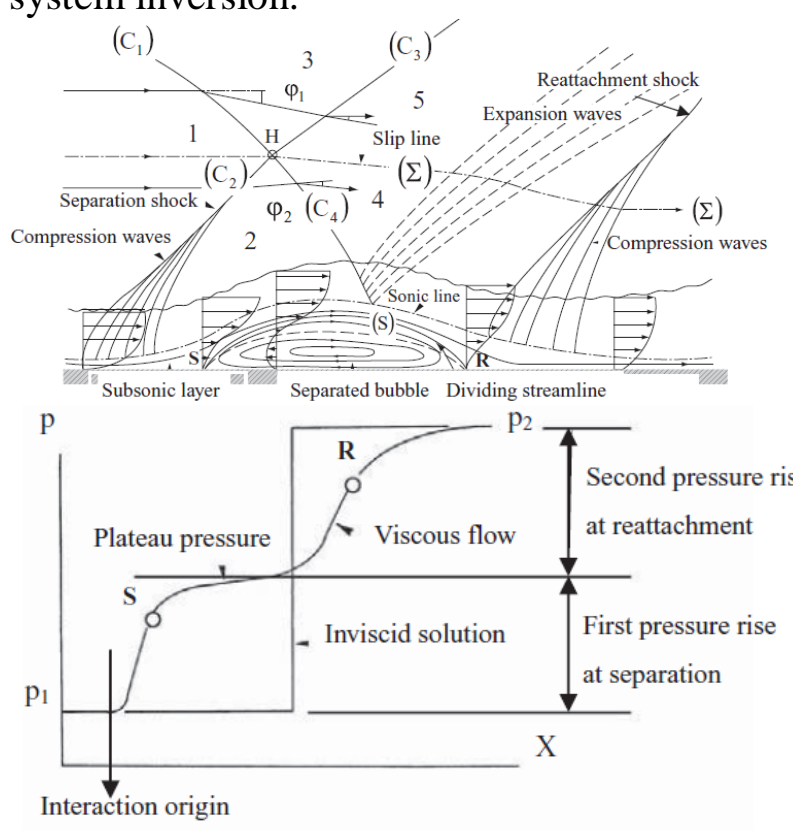

Figure 3 :Schematic physical phenomenon of interaction with separation

\section{Classification of Turbulence Models:}

The classification of Turbulence models i.e Reynolds Averaged Navier strokes has been clearly addressed by Sravankumar Kota et.al[14].Nevertheless, in this paper I have analysed Large eddy simulation model, so superficial information regarding such a model has been following.

\section{Large Eddy Simulation}

Huge Eddy Simulation has totally rely on the probability that two or three sizes of the full stormy approaches are disposed of to get an ideal lessening in the degree of scales required for computational and numerical redirections. Considerably more surely, little sizes of the stream should be ceaselessly exhaustive and less compelled by limit rules than the huge ones in most organizing applications. Enormous augmentations are once in a while moreover not really tended to during the check, their impact should in like way be appeared. Let us first note here that little and immense augmentations are not particularly depicted musings, which are stream subordinate and not precisely coordinated by the authentic hypothesis of LES.In practice, as all amusement methodologies, LES incorporates unraveling the strategy of managing conditions for liquid mechanics (for the most part the Navier-Stokes conditions, possibly redesigned by extra conditions) on a discrete framework, for example utilizing a set number of degrees of possibility. The focal thought is that the spatial course of the structure place focuses verifiably makes a scale portion, since scales littler than a standard scale related to the cross area dispersing can't be gotten. It is in like way admirable seeing that numerical plans used to discretize 
unsurprising directors, since they impel a scale-subordinate mess up, present an extra scale section between especially settled scales and inadequately settled ones.

Therefore, the LES issue make a couple subranges of scales appearing:

- spoke to non-settled scales

- addressed non-settled scales

- non-spoke to scales

The challenging issues in the segment of these computational flows is to grasp and show the nearness of these 3 scale inranges and to make directing conditions for them. To state the exhibiting issue, a couple of numerical approaches for the conclusion of these methods directing conditions initiated in 1973 by professor Leonard, who introduced the isolating thought for clearing little extensions. The isolating thought changes it able to state a couple of issues methodically, including the end issue and the significance of cut-off conditions.

One the other hand the isolating thought presents a couple of old rarities, for instance sensible issues which are missing in the main specifying. A model is the substitution screw up betwixt the round channel and a segregation plot.

The advanced method thought attained in the composition for high computational of compressible streams is the complexity channel method, those are broadly consumed later on. A couple of various thoughts have been proposed for incompressible stream proliferation, most by a long shot of those procured to not in compressible high end flows.

\section{Problem Description}

The geometry, demonstrated schematically in Fig. 4 a, comprises of an Oblique Shock generator model with two distinctive diversion points of 9 and 12 Degrees. The quality of the shock wave increments with flooding the redirection point considerably, bringing about a more grounded association with the limit layer. Free stream conditions are $\mathrm{M} \infty=2.7, \mathrm{~T} \infty$ $=300 \mathrm{~K} \mathrm{~T}($ wall $)=300$ and $\mathrm{p} \infty=100 \mathrm{Kpa}$ with unit Reynolds number Reo $=4300$. The plate is kept up under isothermal states of $300 \mathrm{~K}$. At first, the tests were accomplished for stream over level plate to acquire undisturbed tempestuous limit layer properties like $\delta, \delta+, \theta$ and $\mathrm{Cf}$ at various areas. Divider information like weight, skin grinding and temperature move rates were estimated along the level plate in the connection area.

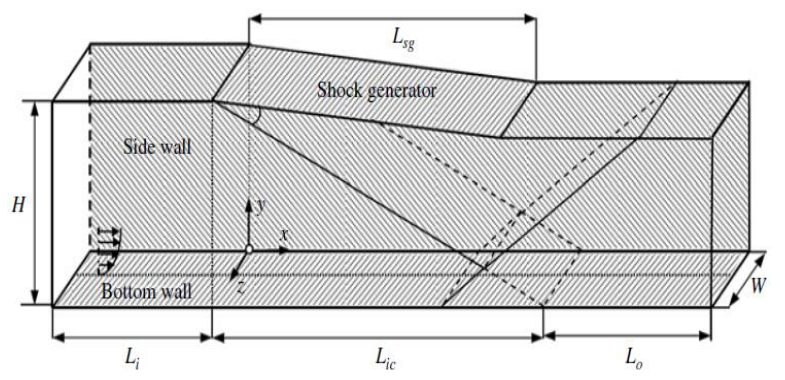

Figure 4: Dimensions and boundary conditions of entire domain.

\section{Computational Domain and Mesh information}

\section{Modelling and Meshing}

Here, I used a tool GAMBIT for modelling and meshing the geometry as per parameters shown in Figure 3.1. During meshing, I have maintained the quality of aspect ratio of 2.15462. As a result, the model attained 390000 hexahedral cells, 1151900 quadrilateral interior faces and 408357 nodes along with Volumes of 25000 as shown in Figure 5. 


\section{Volume statistics:}

minimum volume (m3): 7.883724e-01

maximum volume (m3): $1.000000 \mathrm{e}+00$

total volume (m3): 3.301002e+05

Face area statistics:

minimum face area (m2): 7.883724e-01

maximum face area (m2): $1.000000 \mathrm{e}+00$

Mesh Quality:

Orthogonal Quality ranges from 0 to 1 , where values close to 0 correspond to low quality.

Minimum Orthogonal Quality $=9.87769 \mathrm{e}-01$

Maximum Aspect Ratio $=2.15462 \mathrm{e}+00$

Memory Usage

\begin{tabular}{|l|l|l|l|l|l|}
\hline & Cells & Faces & Nodes & Objps & Edges \\
\hline $\begin{array}{l}\text { Numbers } \\
\text { Used }\end{array}$ & 390000 & 1188100 & 408357 & 13 & 0 \\
\hline $\begin{array}{l}\text { Mbytes } \\
\text { used }\end{array}$ & 298 & 348 & 22 & 0 & 0 \\
\hline $\begin{array}{l}\text { Number } \\
\text { Allocated }\end{array}$ & 390000 & 1188100 & 408357 & 147626 & 0 \\
\hline $\begin{array}{l}\text { Mbytes } \\
\text { Allocated }\end{array}$ & 303 & 356 & 22 & 5 & 0 \\
\hline
\end{tabular}

\section{Analyzing}

Though, there are a lot of CFD analysis software's available for analyzing fluid dynamic models among those FLUENT is the best tool to make analysis easily. The main reason behind choosing fluent is easy to use, Flexibility, Accuracy, allows for efficient execution, interactive control, and complete flexibility, for various operating systems.

\section{CFL Variation}

Numerous Courant-Friedrichs-Lewy (CFL) numbers are used during computations. While computing, it have initiated with CFL of 0.2 is used at the beginning and it is gradually surged to 0.4 in the first 200 iterations. It is further sueged to 1 at 1000 iterations and to 2.0 at 2000 iterations. However, we have not done umpteen iterations unlike 2-Dimensional due to long duration processing computations. The CPU of 27 hours taken to attain 2000 iterations of 25000 volumes.

\section{Boundary Conditions}

In the current examination, an Oblique Impingement SWBLI with a moderate partition is explored, thinking about sidewall impacts. The inflow parameters are set as per the qualities referenced. A full-range stun generator with point 9 and 12 degrees are utilized to create a consistent episode stun with a stun edge of $28: 9$ and a weight proportion of q1.82.

\section{Grid Generation}

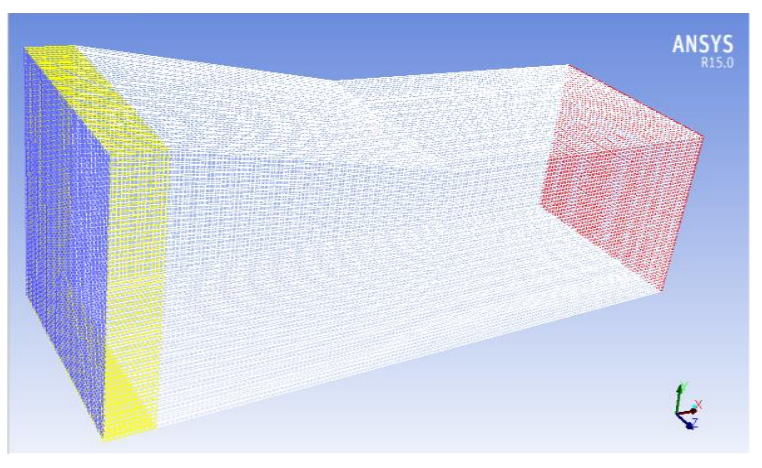

Figure 5: Mesh generation through gambit

\section{Results and Discussion:}

While the assortments in centreline framework implies robust sidewall impacts, the corporeal elaborations for the movements must be established by further examination of the entire 3D stream fields. Snappy and whizz segments for instances of 9 and 12 degrees are detailed in the figure 6 in half of the space (from the centreline to one sidewall). The nearby divider whizz streaks can be doubtlessly found in the fast field showed up in the figure on both sidewall and base divider before the cooperation.

Neighborhood limit layer will also thickening can be viewed, both in the corner and focal point of the territory. The found the center estimation of the speed 
field got in the figure is significantly 3D, with switch stream zones visualizing on the sidewalls in the vicinity of the corner and close the centreline of the space. For the central bifurcation, the close by limit layer thickness with division bubble height both addition towards the central plane. Considering the thickening of the limit layer and the contrary stream zone in the forward segment of the affiliation, the central division will appear to be bowed when seen on a plane at a particular decent route from the divider.

A 3D segment in like manner obtained on the sidewall. Various portions Viz. Z-pivot $0(\mathrm{~m}), 25(\mathrm{~m})$ and $40(\mathrm{~m})$ has been chosen and made investigation over each and singular fragment so as to balance all along with both 9-and 12-degree impingement. Those varieties has been expounded each and singular case with representations separately.

\section{At 9 degrees}

At 9 Degrees, the shock interaction has attained in the position of $83(\mathrm{~m})$ and reattached at 123 in the $\mathrm{X}$-axis, and the viscous layer has formed both left and right walls of the domain. Particularly, the shock intensity has been fierce in both sides. The adverse pressure gradients have obtained more on the sides of the domain to contrast with middle as shown in fig 6 , and the velocity is more in the middle of the domain i.e $25(\mathrm{~m})$. The reflected shock has procured immediately following the interaction towards the upper wall at an angle of 28 Degrees, where as the incident incoming shock angle is 24 Degrees. The maximum pressure Co-efficient has noticed in the middle planar of the entire domain is 0.5 , whereas the normal values has obtained at both left and right walls in between the range of 0.43 to 0.45 . Also, the mach number has slipped from supersonic (2.7) to transonic range through out the shock bubble. Noticeably, the velocity at 0 Metre becomes very low i.e 0.3. Temperature reached maximum exactly at interaction region. We can also observe clearly the shock bubble and the velocity formation through out the domain, along with side walls in figure 7 . All the graphical representation data for afore mentioned has been in the figure 8,9,10.

\section{Wall pressure spectra}

In any case, the computational outcomes do show different practically identical to that observed at the centreline in the equivalent streamwise area as at the inside. In the interim, there is minimal low vitality in the appended narrows betwixt the focal and corner partitions, which may have been normal as detachment is a state of SWBLI flimsiness.

The higher vitality in the corner partitions of experiment may be expected to the the outright tallness of the conduit being multiple times bigger than that of experiment 12 degrees, which may bring about bigger associations of the sidewall limit layers along with the occurrence stun. This theory is bolstered by the obvious thickening of the occurrence stun towards the floor found in the spanwisecoordinated. This is on the grounds that these collaborations are proportional to a cleared SBLI[5]. Consequently, increasingly lively low-recurrence movement may be required because of the bigger partitions. 


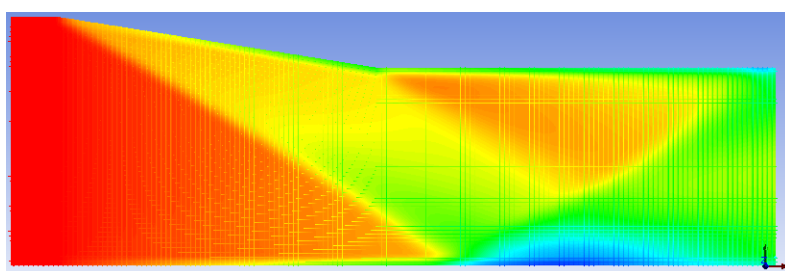

Figure 6: Mach Contours at 9 Degrees

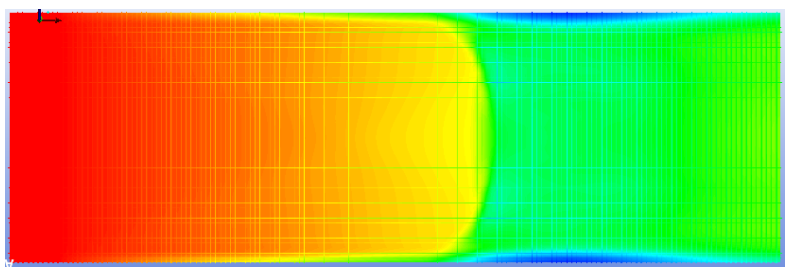

Figure 7: Down Wall at 9 Degrees

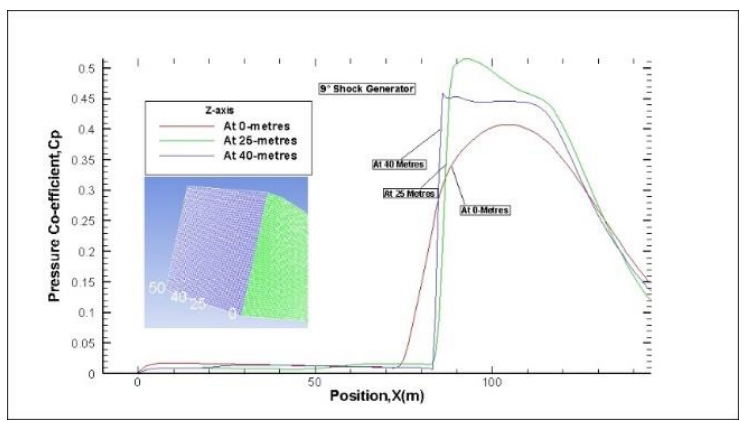

Figure 8: Comparison of Pressure co-efficient at different segments in domain

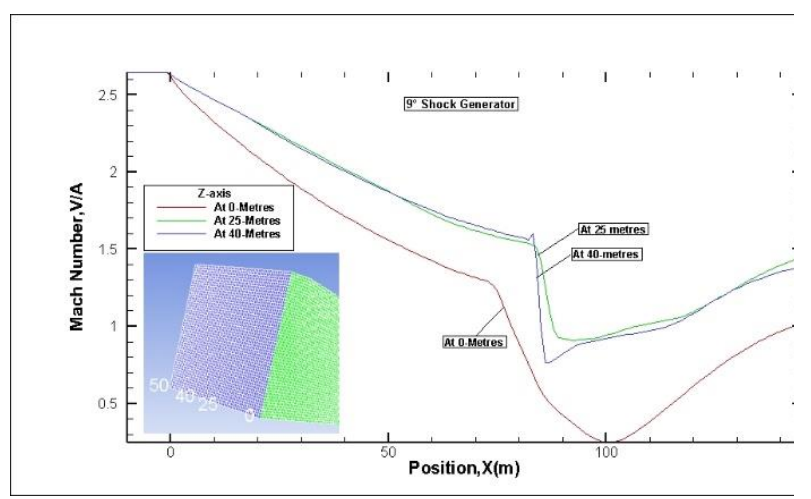

Figure 9: Comparison of Mach Number at different segments in domain

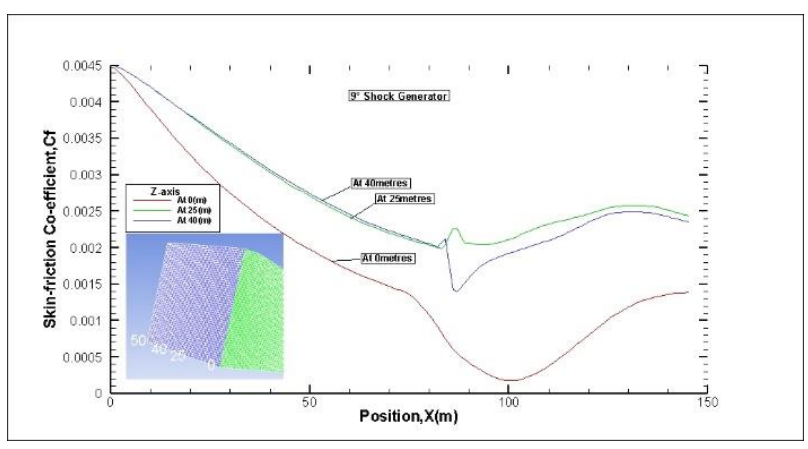

Figure 10: Comparison of Skin friction coefficient at different segments in domain

\section{At 12 Degrees}

At 12 Degrees, the shock interaction has attained in the position of $65(\mathrm{~m})$ and reattached at 96 in the $\mathrm{X}$-axis, and the viscous layer has formed both left and right walls of the domain. Particularly, the shock intensity has been fierce in both sides. The adverse pressure gradients have obtained more on the sides of the domain to contrast with middle, and the velocity is more in the middle of the domain i.e 25(m). The reflected shock has procured immediately following the interaction towards the upper wall at an angle of 24 Degrees, where as the incident incoming shock angle is 22 Degrees. The maximum pressure Co-efficient has noticed in the middle planar of the entire domain is 0.53 , whereas the normal values has obtained at both left and right walls in between the range of 0.48 to 0.47 . Also, the mach number has slipped from supersonic (2.7) to transonic range through out the shock bubble. Noticeably, the velocity at 0 Metre becomes very low i.e 0.025 . Temperature reached maximum exactly at interaction region. Also, viscous wall region has deflecting precisely shown in the interaction region. All the graphical representation data has afore mentioned in the figures 11,12 , and 13 . 


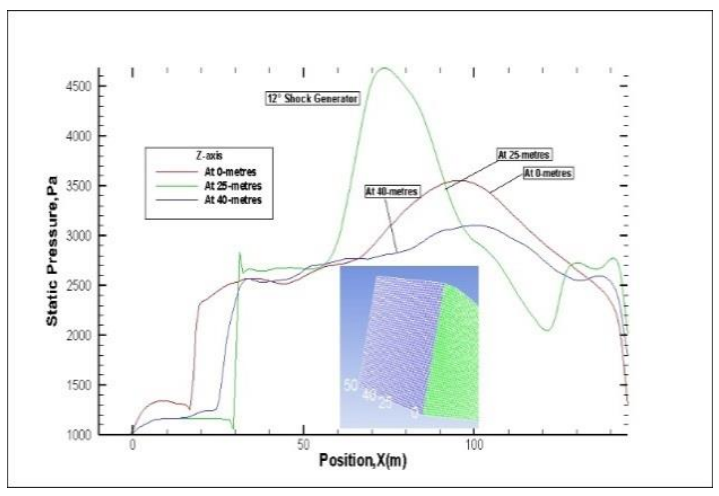

Figure 11: Comparison of Static Pressure at different segments in domain

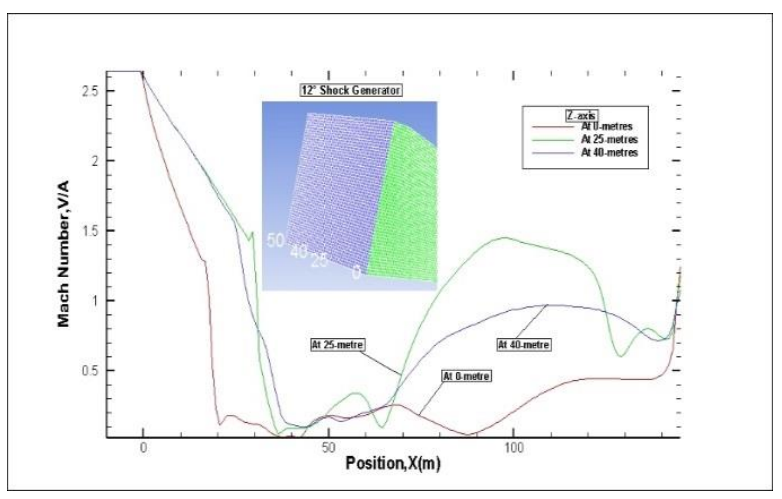

Figure 12: Comparison of Mach Number at different segments in domain

\section{Viscous wall region}

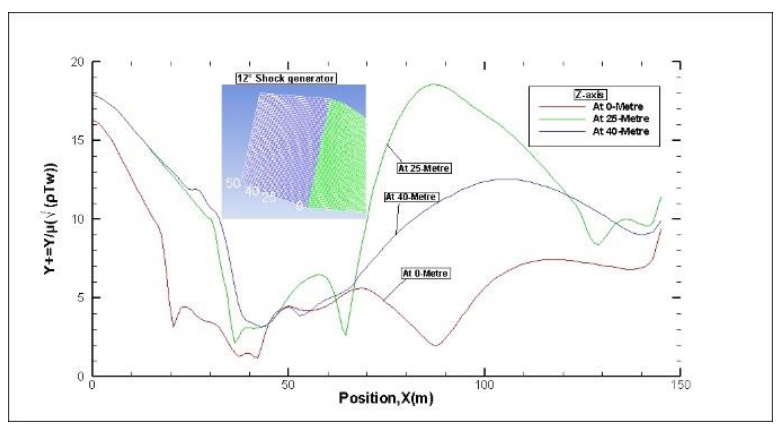

Figure 13: Comparison of Viscosity near Wall at different segments in domain

\section{Conclusion}

Though, analysing Large eddy simulation physical phenomena is being stringent, here the results reveal a efficient data around the domain precisely. To compare with different positions in $\mathrm{X}-\mathrm{Z}$ plane the properties are varying regardless the domain. We clearly notice for changing the flow variation in the vicinity of the walls i.e both left and right, along with the interaction and re-attachment has observed precisely. It is true that the interaction region temperature is being intense for increasing the angle of shock impingement. All the properties are similar in the segment of $0(\mathrm{~m})$ and $40(\mathrm{~m})$ in the domain, where as its has varies in other regions(planar) during interaction region. The re-attachment intense, position of interaction, and size of shock bubble varies to compare with 9 and 12 Degree impingements. In both cases the adverse pressure gradients have procured inside the shock bubble. Flow has transferred to transonic from supersonic in all the cases, which I have done. The skin friction and pressure-coefficient has attained maximum in the region of interaction, and ameliorated immediately after the separation bubble.

\section{Reference}

1.P. K. Rabey, S. P. Jammy, P. J. K. Bruce and N. D. Sandham Two-dimensional unsteadiness map of oblique shock wave/boundary layer interaction with sidewalls by Published online by Journal of Fluid Mechanics Cambridge University Press: 24 May 2019, DOI: https://doi.org/10.1017/jfm.2019.404

2. Holger Babinsky, John K. Harvey, Shock WaveBoundary-Layer Interactions by Cambridge University Press 2011,PP: 31-42

3.Bo Wang1,2, Neil D. Sandham1, †, Zhiwei Hu1 and Weidong Liu2 Numerical study of oblique shock-wave/boundary-layer interaction considering sidewall effects Published online by Journal of Fluid Mechanics Cambridge University Press: 20 February 2015

DOI: https://doi.org/10.1017/jfm.2015.58 
4.A. Hadjadj†, J. Larsson, B. E. Morgan, J. W. Nichols AND S. K. Lele Large-eddy simulation of shock/boundary-layer interaction by Center for Turbulence Research Proceedings of the Summer Program 2010

5.Jian Fang* Beihang Yufeng Yao, Alexandr A. Zheltovodov Khristianovich Lipeng $\mathrm{Lu}$ Investigation of Three-Dimensional Shock Wave/ Turbulent Boundary-Layer Interaction Initiated by a Single Fin by American Institute of Aeronautics and Astronautics Published Online:14 Oct 2016 https://doi.org/10.2514/1.J055283.

6.Garnier, E., Sagaut, P., \& Deville, M. (2002). Large Eddy Simulation of Shock/Boundary-Layer Interaction. AIAA Journal, 40(10), 1935-1944. doi:10.2514/2.1552

7.Yang, G., Yao, Y., Fang, J., Gan, T., \& Lu, L. (2016). Large-Eddy Simulation of ShockWave/Turbulent Boundary Layer Interaction And Its Control Using Sparkjet. International Journal of Modern Physics: Conference Series, 42, 1660186. doi:10.1142/s201019451660186.

8.LOGINOV, M. S., ADAMS, N. A., \& ZHELTOVODOV, A. A. (2006). Large-eddy simulation of shock-wave/turbulent-boundary-layer interaction. Journal of Fluid Mechanics, 565, 135. doi:10.1017/s0022112006000930 .

9.Sandham N.D., Touber E. (2011) Numerical simulations of shock-wave/boundary-layer interaction phenomena. In: Kuerten H., Geurts B., Armenio V., Fröhlich J. (eds) Direct and Large-Eddy Simulation VIII. ERCOFTAC Series, vol 15. Springer, Dordrecht

10.Loginov, M. S., Adams, N. A., \& Zheltovodov, A. A. (2004). Large-Eddy Simulation of Shock Wave/Turbulent Boundary Layer Interaction at High Reynols Number. PAMM, 4(1), 464465. doi:10.1002/pamm.200410213 .

11. Kota, S. K., and Shaik, S. A. (2020). NavierStokes Computations on Type-IVr Shock-Shock Interaction mechanism over Double Wedge (15-60) at High Speed Flows. i-manager's Journal on Mechanical Engineering, 10(1), 21-31.
12.Lusher, D. J., Jammy, S. P., \& Sandham, N. D. (2018). Shock-wave/boundary-layer interactions in the automatic source-code generation framework OpenSBLI. Computers \& Fluids, 173, 1721. doi:10.1016/j.compfluid.2018.03.081.

13.Li, W., \& Liu, H. (2019). Large-eddy simulation of shock-wave/boundary-layer interaction control using a backward facing step. Aerospace Science and Technology, 84, 10111019. doi:10.1016/j.ast.2018.11.005.

14. Sravan Kumar Kota, Mohamaed Shahid, "Linear and Non-Linear Turbulence Models of shock/boundary-layer interaction at Hypersonic Flows," International Journal of Scientific Research in Physics and Applied Sciences, Vol.7, Issue.5, pp.16-25, 2019.

15. Sravan Kumar Kota, P.V Subbaraju "Computational Analysis of Shockwave-Boundary Layer Interaction at Hypersonic Speeds", International Journal of Computer Aided Manufacturing, Vol. 5: Issue 2 Page 4-8,2019. 